\title{
Systemic and environmental risk factors for recurrent aphthous stomatitis in a Polish cohort of patients
}

\author{
Zuzanna Ślebioda, Barbara Dorocka-Bobkowska \\ Department of Gerodontology and Oral Pathology, Poznan University of Medical Sciences, Poznan, Poland \\ Adv Dermatol Allergol 2019; XXXVI (2): 196-201 \\ DOI: https://doi.org/10.5114/ada.2018.74638
}

\begin{abstract}
Introduction: Recurrent aphthous stomatitis (RAS) is a common oral mucosal disease without a clearly defined etiology.

Aim: To analyze the influence of systemic diseases, medications, smoking and a family history of RAS on the prevalence and the course of the condition in Polish patients with RAS.

Material and methods: Seventy-eight patients with RAS and 72 subjects without a history of RAS were enrolled in the study. All participants underwent a detailed oral examination and a full blood count. The results were statistically analyzed with Statistica 10 (StatSoft, Krakow, Poland) with Mann-Whitney, Kruskal-Wallis, $\chi^{2}$ and Fisher tests, with $p<0.05$ considered as the significance level.

Results: The most common systemic conditions observed in patients with RAS were hypertension, allergies and anemia. Both HT and anemia were prevalent in the RAS group. A positive RAS family history occurred more frequently in the study group than in controls. However, no association was found between smoking and RAS prevalence, although a higher percentage of smokers was observed in the RAS subgroup with a low frequency of RAS episodes. Conclusions: The potential role of hypertension and anemia as modifiers of the immune reaction in RAS has been implied in this study but confirmation of this association requires further studies on a larger sample of patients. However, there appears to be no correlation between smoking and the occurrence of RAS. The positive family history observed in this study in the majority of RAS patients confirms the genetic background of the condition.
\end{abstract}

Key words: recurrent aphthous stomatitis, oral pathology, smoking.

\section{Introduction}

Recurrent aphthous stomatitis (RAS) is a common oral mucosal disease of an unknown etiology. Typically lesions present as painful, oval-shaped, single or multiple erosions with an erythematous halo covered by a grey fibro-membranous layer. Based on clinical appearance, three main types of aphthae can be distinguished: major (MaRAS or Sutton's), minor (MiRAS or Mikulicz's) and herpetiform (HeRAS) [1, 2]. Recurrent aphthous stomatitis may appear as an isolated symptom or it may represent an element of systemic syndromes such as Behçet's disease or PFAPA (periodic fever, aphthous stomatitis, pharyngitis and adenitis) [3, 4]. It often accompanies gastrointestinal diseases and immune defects, such as HIV/AIDS [5, 6]. Depending on the origin of the examined subjects and the qualification criteria used, the prevalence of RAS ranges between $5 \%$ and $25 \%$ in the general population with frequency as high as $50-60 \%$ in some selected groups [1, 4]. The onset of ulcers usually occurs during childhood. The condition is most prevalent among individuals in their 20s, with a tendency to diminish in frequency and severity with age. Several studies have reported a higher incidence of RAS among females, non-smokers, white races and people with a high socioeconomic status [4, 7].

The absence of a comprehensive understanding of the etiology of RAS has hindered the treatment of RAS, which is largely empirical and aimed at symptom reduction [7]. However, a disturbed immune response in genetically predisposed subjects has been considered to be an essential factor contributing to the condition. Suggested modifiers include various local and systemic factors, e.g. stress and anxiety, mineral and vitamin insufficiencies, hematologic disorders, food allergies and trauma or even 
handedness [8-12]. Some reports have claimed a lower prevalence of RAS in smokers compared to non-smokers with an increase in the incidence of RAS following smoking cessation, and resolution of lesions upon resumption of cigarette smoking [13-15]. The potential role of systemic diseases and medications as triggers in RAS has also been suggested in several studies, although the results remain equivocal $[16,17]$.

\section{Aim}

In the present study we analyzed the influence of systemic diseases and medications, smoking and positive family history of RAS on prevalence of the disease and the course of the condition in Polish patients with RAS.

\section{Material and methods}

The study group consisted of 78 patients with RAS aged 7-82 years (mean age: 34.5 years) including 31 men aged $7-70$ years (mean age: 35.6 years) and 47 women aged 9-82 years (mean: 33.8 years). The diagnosis of RAS was established from the typical clinical presentation of lesions. All participants underwent a detailed oral examination consisting of anamnesis and clinical evaluation. Age, sex, subjective complaints related to the oral cavity, dental hygiene habits, addictions and RAS family history were included in the patients' history. Clinical evaluations of the oral cavity were performed by a dental specialist qualified in oral pathology and included a detailed assessment of the type, localization and frequency of pathologic lesions. Oral mucosa diseases were diagnosed based on typical clinical symptoms and the results of accessory tests, performed when indicated. Patients with a regular mode of RAS recurrences, defined as 3 episodes in the last 3 years, were enrolled in the study. Detailed information regarding the date of the first episode of RAS, frequency of flare-ups, number of lesions per single flare-up and duration of healing was collected from study group participants. Patients were assigned to MiRAS, MaRAS and HeRAS subgroups in accordance with Stanley's classification [18]. Based on the mode of recurrences, subjects were classified into a mild (episodes with more than 3-month intervals), moderate (episodes separated by intervals between 1 and 3 months), or severe (lesions present almost constantly) RAS course subgroups [19]. According to the number of lesions per single flare-up, the RAS patients were enrolled in group A (1-3 lesions per episode) or group B (over 3 lesions per episode) [12].

The control group comprised 72 subjects with a negative RAS history, aged 1-79 years (mean age: 33.4 years), including 20 men aged $1-70$ years (mean: 33.3 years) and 52 women aged $20-79$ years (mean age: 33.5 years). The controls were recruited from students, staff members and patients. The exclusion criteria for both groups in- cluded the presence of any erosive or ulcerative disease of the oral mucosa revealed during the examination or recorded in the history of the patient and use of immunosuppressive or immunomodulating drugs.

A full blood count with a white blood cell differential count was performed after collecting approximately $10 \mathrm{ml}$ of peripheral blood from all the study subjects and controls.

The study was approved by the Poznan University of Medical Sciences Ethics Committee (approval code: 540/14, with latter changes 868/14) and complied with the guidelines of the Declaration of Helsinki. All the patients were informed in detail about the nature of the study before consent was obtained for the participation in this project.

\section{Statistical analysis}

The results were statistically analyzed with Statistica 10 (StatSoft, Krakow, Poland) for Windows with MannWhitney, Kruskal-Wallis, $\chi^{2}$ and Fisher tests with $p<0.05$ considered as the significance level.

\section{Results}

Table 1 depicts the prevalence of systemic diseases in patients with RAS and in controls.

The most frequent systemic condition observed in patients with RAS was hypertension $(\mathrm{HT})$, reported in $11(14.1 \%)$ cases but present in only three of the controls. The difference between the groups was statistically significant ( $p=0.0367$ ). Allergies to pollen, metals and several food ingredients were diagnosed in 10 (12.82\%) RAS patients and in 7 (9.72\%) controls (statistically insignificant difference; $p=0.5493$ ). Anemia was found in 7 RAS patients and in 1 control $(p=0.0418)$. It included 1 case of microcytic anemia with a decreased serum Fe level, 2 cases of microcytic anemia with a decreased serum vitamin $B_{12}$ and four cases of normocytic anemia. Several diseases were reported only in the study group. That included depression (5 subjects $-6.41 \%$ ), hypercholesterolemia and arrhythmia (3 subjects - 3.85\%) and single cases of diabetes, hyperthyroidism, osteoporosis, epilepsy, psoriasis and cerebral palsy. Bulimia was observed in 1 (1.39\%) control. Diseases of the gastrointestinal tract appeared in 12 (15.38\%) RAS patients and in 5 (6.94\%) controls; the difference between the groups was statistically insignificant ( $p=0.1026)$. Detailed characteristics of the RAS patients suffering hypertension are illustrated in Table 2.

Most of the patients with RAS and hypertension suffered a minor type of RAS (9 subjects, $81.8 \%$ ), while in 2 cases Sutton's (major) aphthae appeared, and was treated with $\beta$-blockers. Over $60 \%$ of RAS patients with HT presented a moderate severity of RAS defined by the frequency of episodes, and in over $90 \%$ of these cases 3 or less lesions formed during one flare-up (group RAS-a). 
Table 1. Systemic diseases in RAS patients and controls based on medical history

\begin{tabular}{|c|c|c|c|c|}
\hline \multirow[t]{2}{*}{ Condition } & \multicolumn{2}{|c|}{ RAS } & \multicolumn{2}{|c|}{ Controls } \\
\hline & $n$ & $\%$ & $n$ & $\%$ \\
\hline Hypertension* & 11 & 14.1 & 3 & 4.17 \\
\hline Allergy & 10 & 12.82 & 7 & 9.72 \\
\hline Hypothyroidism & 7 & 8.97 & 3 & 4.17 \\
\hline Anemia* & 7 & 8.97 & 1 & 1.39 \\
\hline Asthma & 5 & 6.41 & 1 & 1.39 \\
\hline Depression & 5 & 6.41 & 0 & 0 \\
\hline Hypercholesterolemia & 3 & 3.85 & 0 & 0 \\
\hline Arrhythmia & 3 & 3.85 & 0 & 0 \\
\hline Type 2 diabetes & 1 & 1.28 & 0 & 0 \\
\hline Hyperthyroidism & 1 & 1.28 & 0 & 0 \\
\hline Osteoporosis & 1 & 1.28 & 0 & 0 \\
\hline Epilepsy & 1 & 1.28 & 0 & 0 \\
\hline Psoriasis & 1 & 1.28 & 0 & 0 \\
\hline Rheumatoid arthritis & 1 & 1.28 & 1 & 1.39 \\
\hline Cerebral palsy & 1 & 1.28 & 0 & 0 \\
\hline Multiple sclerosis & 1 & 1.28 & 0 & 0 \\
\hline Bulimia & 0 & 0 & 1 & 1.39 \\
\hline Gastrointestinal diseases (in total) & 12 & 15.38 & 5 & 6.94 \\
\hline Peptic ulcer disease & 3 & 3.85 & 0 & 0 \\
\hline Irritable bowel syndrome & 3 & 3.85 & 0 & 0 \\
\hline Gastro-esophageal reflux disease & 3 & 3.85 & 5 & 6.94 \\
\hline Coeliac disease & 3 & 3.85 & 0 & 0 \\
\hline
\end{tabular}

A person who suffered MaRAS with a high frequency of episodes and high number of lesions per 1 flare up was also treated with $\beta$-blockers.

A positive family history (aphthae in at least one firstdegree relative) appeared more frequently in the study group than in controls ( $48.7 \%$ vs. $13.9 \% ; p=0.0001)$. The family history of RAS in the study and control groups is presented in Table 3.

Table 4 depicts the prevalence of smoking in the study group and in controls.

Seventy (89.74\%) RAS patients were non-smokers, while 8 (10.26\%) subjects were smokers. Similar proportions of smokers and non-smokers were observed among the controls. There was no association between smoking and RAS ( $p=0.5577$ ). When comparing the prevalence of smoking in particular subtypes of aphthae (Table 5), an association was found between smoking and the frequency of recurrences $(p=0.0236)$. A higher percentage of smokers was observed in the low-frequency group.
Within RAS group 3, defined as a high-frequency subgroup, none of the subjects was a smoker.

\section{Discussion}

Although the independent risk factors of RAS have not been clearly established in population-based studies, the suggested etiologic factors include genetic mutations, disturbed immune responses, hematinic and zinc deficiencies, gastrointestinal diseases, and defective mucosal epithelial turnover [1, 2, 8, 20]. Individual sources of susceptibility to the disease include various DNA polymorphisms distributed in the human genome, especially those related to alterations in the metabolism of cytokines, e.g. IL-1, II-6 or IL-18 [12, 20]. Another commonly held perception is the inverse relationship between smoking and RAS. Only a few studies have specifically examined this thesis, mostly relying on self-reporting of tobacco habits, which has been shown as an inaccurate method of evaluation [13, 21-24].

In the present study we analyzed the prevalence of systemic diseases, smoking and a positive RAS family history in Polish patients with RAS.

A significantly higher frequency of hypertension and anemia was revealed in RAS subjects than in controls. The severity of RAS in patients with HT was comparable to that observed in all other RAS subjects. Hitherto there have been no reports on the direct association between HT and RAS, although some studies on RAS induced by anti-hypertensives have been published. During the 1970s Seedat described the onset of RAS due to treatment with captopril [25]. Other authors reported the onset of aphthous-like lesions with the use of other $\beta$-blockers, such as labetalol or alendronate [25-27]. Several cases of RAS exacerbation in patients on nicorandil, the K+ATP channel agonist, have been published $[28,29]$. The association between RAS and sartans was first postulated by Goffin et al. In recent years such an association was also suggested for irbesartan [30, 31]. However, in a case-control study by Boulinguez et al. only an association between $\beta$-blockers and RAS could be confirmed $(p=0.002)$ [17]. In the present study several types of anti-hypertensives were used in the RAS group, but the most severe course of the disease was found in subjects using $\beta$-blockers. This occurred in $45.5 \%$ of the study participants with HT aphthae that developed at the age over 40 years, which is not a typical age for the onset of RAS. This may suggest the development of aphthouslike lesions induced by anti-hypertensives.

Allergies were another common systemic disorder in our study group, mainly due to pollen, dust mites, and some food ingredients. However, the prevalence of allergies in the study and control groups was comparable, excluding an association with RAS. A potential role of allergies in the development of RAS was suggested in some reports. The presence of allergens could initiate the cyto- 
Table 2. Clinical characteristics of RAS patients with hypertension

\begin{tabular}{cccccccc}
\hline No. Gender & Age & Antihypertensive drug & $\begin{array}{c}\text { Age of } \\
\text { RAS } \\
\text { onset }\end{array}$ & $\begin{array}{c}\text { RAS } \\
\text { clinical } \\
\text { type }\end{array}$ & $\begin{array}{c}\text { RAS classification } \\
\text { according to } \\
\text { episodes' frequency }\end{array}$ & $\begin{array}{c}\text { RAS classification } \\
\text { according to number } \\
\text { of lesions per flare up }\end{array}$ \\
\hline 1 & $\mathrm{~F}$ & 24 & None & 20 & MiRAS & RAS-2 & RAS-a \\
\hline 2 & $\mathrm{~F}$ & 52 & Valsartan (AT2 receptor antagonist) & 35 & MiRAS & RAS-1 & RAS-a \\
\hline 3 & $\mathrm{~F}$ & 82 & Bisoprolol ( $\beta$-blocker) & 30 & MaRAS & RAS-2 & RAS-a \\
\hline 4 & $\mathrm{~F}$ & 58 & No data & 54 & MiRAS & RAS-2 & RAS-a \\
\hline 5 & M & 66 & $\begin{array}{c}\text { Perindopril (AT2 receptor inhibitor) } \\
\text { furosemide (sulfonamide) }\end{array}$ & 62 & MiRAS & RAS-2 & RAS-a \\
\hline 6 & M & 44 & Perindopril (AT2 receptor inhibitor) & 42 & MiRAS & RAS-2 & RAS-a \\
\hline 7 & F & 51 & Indapamide (sulfonamide) & 45 & MiRAS & RAS-2 & RAS-a \\
\hline 8 & M & 57 & Lisinopril (ACE inhibitor) & 25 & MiRAS & RAS-1 & RAS-a \\
\hline 9 & $\mathrm{~F}$ & 49 & No data & 20 & MiRAS & RAS-1 & RAS-a \\
\hline 10 & $\mathrm{~F}$ & 46 & Nebivolol ( $\beta$-blocker) & $<20$ & MaRAS & RAS-3 & RAS-b \\
\hline 11 & $\mathrm{~F}$ & 71 & Captopril (ACE inhibitor) & 40 & MiRAS & RAS-2 & RAS-a \\
\hline
\end{tabular}

MiRAS - minor aphthae, MaRAS - major aphthae, RAS-1 - low severity of RAS, RAS-2 - moderate severity of RAS, RAS-3 - high severity of RAS, RAS-a - low number of lesions per flare up, RAS-b - high number of lesions per flare up, AT2 - angiotensin II, ACE - angiotensin-converting enzyme.

Table 3. Family history of RAS in study group and controls

\begin{tabular}{lccccc}
\hline Group & \multicolumn{2}{c}{ “+” family history } & \multicolumn{2}{c}{ “-” family history } & \multirow{2}{*}{ P-value } \\
\cline { 2 - 5 } & $n$ & $\%$ & $n$ & $\%$ & \\
\hline RAS & 38 & 48.7 & 40 & 51.3 & \multirow{2}{*}{$0.0001^{*}$} \\
\hline Control & 10 & 13.9 & 62 & 86.1 & \\
\hline
\end{tabular}

*Statistically significant.

kine cascade leading to the formation of aphthae. Milk antigens and gluten were propounded among potential food ingredients triggering the RAS exacerbations [32].

We found a significantly higher frequency of anemia among the RAS patients than in the controls (7, 8.97\% vs. 1,1.39\%). Similar prevalence of anemia among the people with RAS was reported by Burgan et al., who found it present in $14 \%$ of the study participants with RAS. However there was no significant difference between RAS and controls [33], unlike the findings of Compilato et al. (344\% vs. 6.9\%) [34]. Rogers et al. found anemia in only $5.9 \%$ of RAS patients, while Thongprasom et al. and Olson et al. did not report any case of anemia in their studied RAS population [35-37].

We did not observe a higher prevalence of gastrointestinal ( $\mathrm{Gl}$ ) diseases among the RAS patients in this study despite some reports of common GI tract disorders which accompany RAS, including coeliac disease and inflammatory bowel diseases [5]. Based on our own previous observations, RAS was found significantly more often among the patients with Crohn's disease than in generally healthy controls (27.1\% vs. $8.6 \%$ ) [38]. The relationship between gastrointestinal disturbances and RAS may at least be partially due to disturbed absorption of microelements, including iron, vitamins and folate. Con-
Table 4. Prevalence of smoking in study group and controls

\begin{tabular}{lccccc}
\hline \multirow{2}{*}{ Group } & \multicolumn{2}{c}{ Non-smokers } & \multicolumn{2}{c}{ Smokers } & \multirow{2}{*}{$P$-value } \\
\cline { 2 - 4 } & $n$ & $\%$ & $n$ & $\%$ & \\
\hline RAS & 70 & 89.74 & 8 & 10.26 & \multirow{2}{*}{0.5577} \\
\cline { 1 - 4 } Control & 67 & 93.06 & 5 & 6.94 & \\
\hline
\end{tabular}

Table 5. Prevalence of smoking in RAS subtypes

\begin{tabular}{|c|c|c|c|c|c|}
\hline \multirow[t]{2}{*}{ RAS type } & \multicolumn{2}{|c|}{ Smokers } & \multicolumn{2}{|c|}{ Non-smokers } & \multirow[t]{2}{*}{$P$-value } \\
\hline & $n$ & $\begin{array}{l}\% \text { of RAS } \\
\text { subtype }\end{array}$ & $n$ & $\begin{array}{l}\% \text { of RAS } \\
\text { subtype }\end{array}$ & \\
\hline MiRAS & 7 & 11.67 & 53 & 88.33 & \multirow[t]{2}{*}{0.4535} \\
\hline $\begin{array}{l}\text { MaRAS + } \\
\text { HeRAS }\end{array}$ & 1 & 5.56 & 17 & 94.44 & \\
\hline RAS-1 & 6 & 23.08 & 20 & 76.92 & \multirow[t]{3}{*}{$0.0236^{\star}$} \\
\hline RAS-2 & 2 & 6.25 & 30 & 93.75 & \\
\hline RAS-3 & 0 & 0 & 20 & 100 & \\
\hline RAS-a & 8 & 12.70 & 55 & 87.30 & \multirow[t]{2}{*}{0.3417} \\
\hline RAS-b & 0 & 0.00 & 15 & 100.0 & \\
\hline
\end{tabular}

MiRAS - minor aphthae, MaRAS - major aphthae, RAS-1 - low severity of RAS, RAS-2 - moderate severity of RAS, RAS-3 - high severity of RAS, RAS-a - low number of lesions per flare up, RAS-b-high number of lesions per flare up. *Statistically significant.

sidering the ambiguous results from these studies, the value of performing routine screening of RAS patients for coeliac disease remains questionable.

The results of our study confirm the high prevalence of a positive family history of the disease. At least one first-degree relative suffered RAS in $48.7 \%$ of the pa- 
tients from the study group. By comparison, a positive family history of RAS was reported by $13.9 \%$ of the controls. However, no association between a positive family history and the severity of the diseases was observed. A high prevalence of a positive family history in RAS was reported by Compilato et al., who also found an earlier onset and a more severe course of RAS in patients with a positive family history [34]. Similar observations were presented by Lopez-Jornet et al., who estimated the risk of RAS in a child of two parents affected with RAS at $67-90 \%$ and $40 \%$ when only one parent suffered from RAS [39]. A correlation between RAS and monozygotic twins was also reported [40]. A significant role of genetic predisposition in the etiology of RAS seems to be certain.

The prevalence of smoking in our study group was comparable to controls. There was however a negative association between smoking and the frequency of RAS recurrences. All of the subjects from the RAS-3 group, where the recurrences were most frequent, were nonsmokers. Numerous literature reports indicate a lower prevalence of RAS among smokers, an inverse correlation between smoking intensity and the severity of RAS, or the onset of RAS after smoking cessation [7, 13, 24]. The less intense course of RAS in smokers may result from hyperkeratinization of the oral mucosa that acts protectively against mechanical and chemical injuries. Exposure to nicotine may modify the local immunologic response and influence the severity of RAS in this manner [13, 24].

The number of controlled studies analyzing systemic conditions and habits in RAS patients is still very limited. Despite the equivocal reports on this subject, our study results indicate an association between hypertension, anemia, and positive family history in a sample of a Polish population. Considering several limitations of this study including the restricted study period and high heterogeneity of the tested group, more definitive results could be obtained from a longitudinal study with a larger patient group. As the poorly understood RAS etiology hinders formulation of an effective treatment regimen, further observations are definitely required to clarify the relevance of local and systemic factors in RAS etiology and its potential impact on the patient's clinical phenotype.

\section{Acknowledgments}

The authors gratefully acknowledge Dr hab. Elżbieta Szponar for her contribution to the study design and Dr n. med. Ewa Krawiecka for her assistance in the data collection.

\section{Conflict of interest}

The authors declare no conflict of interest.

\section{References}

1. Ship JA. Recurrent aphthous stomatitis. An update. Oral Surg Oral Med Oral Pathol Oral Radiol Endod 1996; 81: 141-7.
2. McCullough MJ, Abdel-Hafeth S, Scully C. Recurrent aphthous stomatitis revisited; clinical features, associations, and new association with infant feeding practices? I Oral Pathol Med 2007; 36: 615-20.

3. Baş Y, Seçkin HY, Kalkan G, et al. Investigation of Behçet's disease and recurrent aphthous stomatitis frequency: the highest prevalence in Turkey. Balkan Med J 2016; 33: 390-5.

4. Rivera-Hidalgo F, Shulman JD, Beach MM. The association of tobacco and other factors with recurrent aphthous stomatitis in an US adult population. Oral Dis 2004; 10: 335-45.

5. Yazdanbod A, Nemati R, Iranparvar Alamdari M, et al. Prevalenece of celiac disease in patients with recurrent aphthous stomatitis. Govaresh 2014; 18: 246-51.

6. Miziara ID, Araujo Filho BC, Weber R. AIDS and recurrent aphthous stomatitis. Braz J Otorhinolaryngol 2005; 71: 517-20.

7. Chattopadhyay A, Chatterjee S. Risk indicators for recurrent aphthous ulcers among adults in the US. Community Dent Oral Epidemiol 2007; 35: 152-9.

8. Koybasi S, Parlak AH, Serin E, et al. Recurrent aphthous stomatitis: investigation of possible etiologic factors. Am J Otolaryngol 2006; 27: 229-32.

9. Kunikullaya UK, Kumar MA, Ananthakrishnan V, Jaisri G. Stress as a cause of recurrent aphthous atomatitis and its correlation with salivary stress markers. Chin J Physiol 2017; 60: 226-30.

10. Ciçek Y, Canakçi V, Ozgöz M, et al. Prevalence and handedness correlates of recurrent aphthous stomatitis in the Turkish population. J Public Health Dent 2004; 64: 151-6.

11. Gavic L, Cigic L, Biocina Lukenda D, et al. The role of anxiety, depression, and psychological stress on the clinical status of recurrent aphthous stomatitis and oral lichen planus. J Oral Pathol Med 2014; 43: 410-7.

12. Guimarães AL, De Sá AR, Victória JM, et al. Association of interleukin-1beta polymorphism with recurrent aphthous stomatitis in Brazilian individuals. Oral Dis 2006; 12: 580-3.

13. Axéll T, Henricsson V. Association between recurrent aphthous ulcers and tobacco habits. Scand I Dent Res 1985; 93 : 239-42.

14. Subramanyam RV. Occurrence of recurrent aphthous stomatitis only on lining mucosa and its relationship to smokinga possible hypothesis. Med Hypotheses 2011; 77: 185-7.

15. Marakoğlu K, Sezer RE, Toker HC, Marakoğlu I. The recurrent aphthous stomatitis frequency in the smoking cessation people. Clin Oral Invest 2007; 11: 149-53.

16. Hasan AA, Ciancio S. Association between ingestion of nonsteroidal anti-inflammatory drugs and the emergence of aphthous-like ulcers. J Int Acad Periodontol 2009; 11: 155-9.

17. Boulinguez S, Reix S, Bedane C, et al. Role of drug exposure in aphthous ulcers: a case-control study. Br J Dermatol 2000; 143: 1261-5.

18. Stanley HR. Aphthous lesions. Oral Surg Oral Med Oral Pathol 1972; 33: 407-16.

19. Bagan JV, Sanchis JM, Milián MA, et al. Recurrent aphthous stomatitis. A study of the clinical characteristics of lesions in 93 cases. J Oral Pathol Med 1991; 20: 395-7.

20.Ślebioda Z, Szponar E, Linke K. Stan błony śluzowej jamy ustnej u osób dorosłych z choroba Leśniowskiego-Crohna w zależności od aktywności choroby, metod jej leczenia i palenia papierosów. Prz Gastroenterol 2011; 6: 97-101.

21. Atkin PA, Xu X, Thornhill MH. Minor recurrent aphthous stomatitis and smoking: an epidemiological study measuring plasma cotinine. Oral Dis 2002; 8: 173-6.

22. Little SJ, Stevens VJ, LaChance PA, et al. Smokeless tobacco habits and oral mucosal lesions in dental patients. J Public Health Dent 1992; 52: 269-76. 
23. Pérez-Stable EJ, Marín G, Marín BV, Benowitz NL. Misclassification of smoking status by self-reported cigarette consumption. Am Rev Respir Dis 1992; 145: 53-7.

24.Tüzün B, Wolf R, Tüzün Y, Serdaroğlu S. Recurrent aphthous stomatitis and smoking. Int I Dermatol 2000; 39: 358-60.

25. Seedat YK. Aphthous ulcers of mouth from captopril. Lancet 1979; 15: 1297-8.

26. Pradalier A, Dry J, Baron JF. Aphthoid stomatitis induced by labetalol. Therapie 1982; 37: 695-7.

27. Scully C, Bagan JV. Adverse drug reactions in the orofacial region. Crit Rev Oral Biol Med 2004; 15: 221-40.

28. Healy CM, Smyth Y, Flint RS. Persistent nicorandil induced oral ulceration. Heart 2004; 90: e38.

29. Farah CS, Carey LM, Savage NV. Nicorandil induced oral ulceration. Aust Fam Physician 2003; 32: 452-3.

30.Goffin E, Pochet JM, Lejuste P, De Plaen JF. Aphtous ulcers of the mouth associated with losartan. Clin Nephrol 1998; 50: 197.

31. Manunza F, Atzori L, Pilloni L, Ferreli C. Irbesartan-induced aphthous stomatitis. Glob Dermatol 2015; 2: 62-3.

32. Wray D. Gluten-sensitive recurrent aphthous stomatitis. Dig Dis Sci 1998; 26: 737-40.

33. Burgan SZ, Sawair FA, Amarin ZO. Hematologic status in patients with recurrent aphthous stomatitis in Jordan. Saudi Med J 2006; 27: 381-4.

34. Compilato D, Carroccio A, Calvino F, et al. Haematological deficiencies in patients with recurrent aphthosis. J Eur Acad Dermatol Venereol 2010; 24: 667-73.

35. Rogers III RS, Hutton KP. Screening for haematinic deficiencies in patients with recurrent aphthous stomatitis. Aust J Derm 1986; 27: 98-103.

36. Thongprasom K, Youngnak P, Aneksuk V. Hematologic abnormalities in recurrent oral ulceration. Southeast Asian J Trop Med Public Health 2002; 33: 872-7.

37. Olson JA, Feinberg BA, Silverman S, et al. Serum vitamin B, folate, and iron levels in recurrent aphthous ulceration. Oral Surg 1982; 54: 517-20.

38. Ślebioda Z, Krawiecka E, Rozmiarek M, et al. Clinical phenotype of recurrent aphthous stomatitis and interleukin-1beta genotype in a Polish cohort of patients. J Oral Pathol Med 2017; 46: 657-62.

39. Lopez-Jornet P, Camacho-Alonso F, Martos N. Haematological study of patients with aphthous stomatitis. Int J Dermatol 2014; 53: 159-63.

40. Miller MF, Garfunkel AA, Ram C, Ship II. Inheritance patterns in recurrent aphthous ulcers: twin and pedigree data. Oral Surg Oral Med Oral Pathol Oral Radiol Endod 1977; 43: 886-91. 